\title{
Index
}

absolute value $196,202,203$ acceleration principle 126 , $183,184,186,189,192$, 193

Afghanistan 7, 12, 75

Africa $11,32,75-77$

aggression 12,18

agriculture $30,31,46,47,56$, $58,102,167,180$

AIC 204

Air Pollution Prevention and

Control Law 103, 168

American economy 5, 10,71, $125,144,164$

American financial institutions 5, $6,26,73,134$

An Ziwen 118, 121

Anderson, Jonathan 80

Asia $3,11,22,65,77,86$, $114,117,199,202,206$, 209

atomic energy $\quad 103$

autoregression coefficient 205

Aynak 75

barefoot doctor 99,100

barters 49
Beijing $9,39,42,50,52,58$, $102,115,125,134,156,161$, $167,177,180$

Beijing 2008 Olympics 180

billionaire 20,21

bio-diesel 179

bio-ethanol 179

biogas 179,180

Biographies of the Money Markets 75

biological systems 102

birth rate $36,165,166$

Brazil 79-81, 85

Buffet, Warren 20

BYD Auto 20

Canada 75

carbon dioxide 104, 171, 174

censorship 51

Central Bank Law 40

Central Committee of the

Communist Party 30, 50

central government 17, 18,

25, 37, 47, 48, 54, 96-101,

103-105, 123, 124, 160,

$168,171,172,174,175$,

177 
central planning $8,17,29,45$,

49, 53, 89-91, 126, 147, 149,

155,161

chemical products 30

Chiang Kai-shek 89, 117

Chile 59

China

bureaucracy $6,16,63,68,72$, 95

capitalism 25, 114

diplomacy 75,81

GDP $4,5,8,32-34,59,64$, $85,99,104,144,161$, 170-172, 184, 185, 190, 192,195

urban housing $112,128,134$, 144

China Metallurgical Group Corp 75

China model 7

China's Investment Corporation 80

Chinese Communist Party 7, 49, 66,73

Chinese Constitution 15,50 , 96

Chinese consumers 6, 72, 134, 137

Chinese Economists Society 39, 44,107

Chinese entrepreneurs $6-8,11$, $20,21,25-27,72,73,174$

Chinese financial institutions 6 , $72,73,134$

Chinese Government $4,5,7-13$, $15,17-19,25,26,30,32,36$, $37,40,42,45,46,49,52,54$, $55,63-65,67,68,73,82,84$,
$101,103,106,116,134,138$, $140,159,162,164,168,170$, $171,174-177,180,192$

Chinese history 21, 39

Chinese society $22,37,49,138$

Chinese tourists 5

Chow, Gregory C 80

civil war $16,22,64$

Clean Development Mechanism 179

Cleaner Production Promotion Law 169

climate change $102,169,179$

$\mathrm{CO}_{2}$ emission $102,169,178$

coal $30,103,167,173-175$, 179,180

coastal provinces 4,27

Cobb-Douglas production function 113,126

coercion $48,52,53$

Collective Medical System (CMS) 100

collectivism $6,37,38,72$

Columbia 42

commerce 30

Commercial Bank Law 40

consumer goods 5, 46-49, 101, $133,134,144$

Commission for Reconstructing the Economic System 17, 39, 118

commune system $46,48,53,99$, 100,123

communication $30,90,102$, 122

Communist Party of China (CPC) 15,16

compensation and damage 62 
competition $8,11,38,42,47$, $66,68,101,128,148,157$

Confucian ethics 72

Confucianism 6, 72

Constitution 15, 50, 96, 103, 168,172

constrained maximization 112 , 113

consumer products 30,68

consumption

domestic consumption 9, 137, 138,162

consumption function 113,184 , 186, 192

Copenhagen 169, 171

Cornell 80

Corporate Average Fuel

Efficiency(CAFE) 170

correlation $193,202,203$

corruption $7,19,49,64,73,84$,

$86,95,96,105,157$

coupons 46

covariance matrix of returns

200

credit card $5,6,72,129$

Cultural Revolution 16, 18, 21, 116

culture $21,22,26,27,31,73$, 152

Cyber City 83

death rate 100

Brautigam, Deborah 76

democracy $11,19,31,67,76$,

84,90

democratic government 7,48 ,

66-68, 76, 114, 177

demographer 165
Deng Xiaoping $16,27,46,56$,

$65,127,159$

derivative securities 26

desired capital stock 183

determinant $113,185,190$

development $3,6-8,10,11,15$, $16,19,21-23,29-32,34,35$,

$37,39-42,44,45-55,59,64$, $68,72,76,79,83,84,86,87$, 89-91, 95, 96, 99, 101, 107, $109,110,114-116,123,124$, $139,140,147,149,152,155$, $157,159,163,167-175$, $177-180,195,200,202$

developing country $22,68,83$, 114

Dongfeng Automobile Co. 180 down payment $5,6,72,133$,

134

Dragon's Gift:The Real Story of China in Africa 76

Dybvig, Phil 159

Dynamic Optimization 121, 122

Eastern Europe $17,119,127$

econometric model 115, $121-123,200$

economic advice $111,117,119$, 123

economic behavior $25,27,36$, 108, 109

economic downturn $5,7,26$, $71-73,133,134,137$

economic forecast 115

economic freedom 18, 45, 47,

$48,50,51,66$

economic growth $3,4,7,9,11$, $21,66,73,80,83,85-87$, 
$105,113-116,126,128,144$, $157,162,169,172$

economic incentives 36,104 ,

170,173

economic institution $1,6,17$,

$26,42,64,71,87,109,128$,

129,134

economic planning

scope $29,31,152,170$

economic policy 29, 80, 111,

$117,119-122$

economic power $5,8,11,12,19$,

$48,49,66,95,96,129$

economic reform

dual price system 128

gradual and experimental approach 47

reform of economic institutions 64

Economic Reform Commission

$17,118,120,121$

economic rent 25

economic science 111,112

economic teaching and research

41

economic zones 16,127

economics $3,17,18,41-44,50$,

$58,77,79-81,107-110,112$,

$114,117,119,120,121$,

144, 158-160, 189, 194,

196, 199

education

compulsory education 98

demand $10,47,51,58,65$, $66,75,95,101,102,108$, $112,118,125,134,144$, $148,149,164,173,176$, 197 higher education $32,55,56$,

$58,60,68,79,99,147,149$

private education 23,59

university education 147 , 149

efficiency $16,18,19,22,36$, $107,128,155,157,170$

Egypt 76

Einstein, Albert 139

elasticities of demand 112

election

direct elections of village officials 48

electric power 30

electronics 30

EMBA programs 43

emerging market economies 79,

85

energy $5,11,30,31,33,34$, 102-104, 109, 168, 170-176, 178-180

Energy Conservation Law 103, 168

energy shortage 102

entrepreneurs $3,6-8,11,15$, 19-22, 25-27, 43, 72, 73, 84, $86,91,115,174$

entrepreneurship $4,21,66,87$, 90

environmental degradation 95, 102

environmental protection 30,35 , $65,103-105,109,168-172$, $175-177,180$

error correction model 113, 126

Europe 11, 17, 75, 173

exchange rate $9-11,77,118$,

$129,162-164$ 
executive order 101

exogenous 184,193

expansion of credit 64

exports $5,9,46,62,79,80$,

$137,138,144,162,183-185$,

190, 192

Fanny May 134

Fair, Ray 122

Federal Reserve 10, 45, 164

finance $30,53,60,64,71,80$, $99,101,108,120,158-160$, 178

financial economics 108,112

financial capital 200

financial crisis $26,65,71,72,76$, $80,114,117,129,134,196$,

197, 199, 201, 202, 206-208, 210

financial derivatives 5,71

First Solar 20, 173

fiscal policies 36

fisheries 30

Five-Year Plan

$10^{\text {th }}$ Five Year Plan 174

$11^{\text {th }}$ Five-Year Plan 31-33, $170,172,174,175,179$

$12^{\text {th }}$ Five-Year Plan $10,31,32$, $34,138,163$

fluctuations 29,144

Forbes 20, 21

foreign assets 8

foreign competition 47,66

foreign economic assistance 5

foreign investment $5,27,46,48$, $59,76,79,127-129,200$

foreign reserves 10, 128, 163, 164 foreign scholars 42

foreign trade $9,46-48,65,77$, $128,129,162$

forestry 30

"Four Dragons in Asia" 22

Free to Choose $42,45,47,51$, $52,60-63,66,138$

freedom of speech 50

freedom of the press 19,50

G2 4

GARCH models 200

Gates, Robert 12, 77

GDP

China 4

US 4,5

General Administration of Quality

Supervision, Inspection and

Quarantine 62

General Secretary 18, 49, 67

Germany 22, 114, 169

Getulio Vargas Foundation (GVF)

79

global recession 32

global warming 102

globalization $11,12,38,42$,

144, 195, 197, 200, 201,

210

government activities 37

government agencies 36,67

government bureaucrats 48,72 ,

96

government expenditures 7,32 ,

$36,65,134,143,189,192$

government intervention 6,61 ,

72

government procurement agency

46 
government procurement and trading 48

government protection 63

governmental subsidies 59,60 ,

174

Great Depression $109,134,143$

Great Leap Forward 8, 16, 21, 64

greenhouse gas 172

growth 3, 4, 6-9, 11, 21, 29, 65, $66,72,73,80,83,85-87$, $101,105,113-116,126,128$, $137,144,157,161,162,166$, $169,172,192,197$

Guangzhou 18, 52, 58, 139, $140,157,159$

Guangzhou Opera House 139, 140

Guanxi 26, 114

Gurgaon 83,84

Hadid, Zaha 139

Hall, Robert 183,189

Han Dynasty 22

Hang Seng Index 116

Hangzhou 17

harmonious society 31,157

Harvard 42

health care

demand $10,47,51,58,65$, $66,75,95,101,102,108$, $112,118,125,134,144$, $148,149,164,173,176$, 197

hospital bed 101 local clinics 99, 100 medical services 55 number of doctors 101 public hospital 101 public supply 101 supply $8-10,36,47,64-66$, $68,80,95,101-103,108$, $112,113,115,118,128$, $143,144,148,149$, $161-164,167,179$

history $21,22,29,39,76,84$, $89,109,143,167,201$

Hong Kong 3, 16, 20-22, 27, $46,51,59,83,86,113,114$, $116,121,137,140,148,152$, $153,157,176,178,196$

Hong Kong University of Science and Technology 176, 178 household responsibility system $46,48,125$

housing bubble $5,71,133,134$, 163

Houthakker, H. S. 125

Hu Jintao 67, 169

Huang Yasheng 80

human capital 3, 4, 21, 22, 32,

$83,84,86,87,114-16,138$

human rights $7,11,76$

hybrid vehicle 20

hydropower 103,179

illegal levies 98

imports 5, 10, 46, 128, 138,

$164,174,183-185,190$

inadequate financing 99

income disparity $32,95,97-99$, 105

income per capita 33

India $3,27,79,83-86,144$

indirect election process 18,26

individual freedom 38 
individualism $6,37,72$

inequality $38,99,140$

inflation $8-10,38,63-65,109$,

$113,115,121,161-164,196$, 197

infrastructure investment 197 innovations 4, 42, 62, 63, 68, 110,124

institutional difference 71,112 , 113

intellectual property rights 52

international consensus 104

International Monetary Fund 4, 117

Interpreting China's Economy 109

investment

aggregate investment 126, 183,189

investment abroad $5,8,11$

investment function 113,186

invisible hands 61

Iran 75,76

Iraq $7,12,75,76$

iron ore 79,80

Japan $3,12,17,22,83,86,102$, 114

Jeffersonian doctrine 6,72

Jiang Zemin 49

Jin Liqun 80

job security 53,68

Journal of Comparative Economics 199

Kalman filter 205

Kazakstan 75

Keynes' General Theory 109
Keynesian fiscal policy 68

Kuznets curve 173

Kyoto 103, 169

Kyoto Protocol 169

lag variable 208

lagged consumption $\quad 186$

lagged return 200, 203

Lardy, Nicholas 80

law enforcement 86,104

Law on the Protection of the

Rights and Interests of

Consumers 61

legal institutions 26,87

leisure time 112

Li Karshing 21

Libya 76

life expectancy 100

Lingnan University 159

Liu Hongru 121

Liu Changjiang 199

Liu, T C 117, 121, 123

LNG(Liquefied Natural Gas)

180

loan $72,133,171,173$

low-carbon technology 171

Lucas, Robert 121

machine building 30

macro-economic policy 29

macro-economy 19

macroeconomics $108,112,113$

magnitude 112

manufacturing 47,90

Mao Zedong 16

market economy $3,6,7,19,21$, $22,25,29-32,39,40,47,49$, $58,66,68,71-73,83-85,90$, 
$107,114,119,124,127,143$, $144,147,155$

market forces 66,100

market institutions $3-5,7,19,25$, $37,39,53,68,71,73,86,87$, $89,91,92,112,114,116,127$ Marx, Karl 125 mass infrastructure 32

Maximization $27,112,113$

Maximum Likelihood

Estimation(MEL) 205

MBA 148

media $40,67,98$

medical insurance 55,123

Mei Jianping 80

metallurgy 30

method of production 103

microeconomics $108,112,113$

Middle East $32,76,77$

migrant workers 97

military power 12

Milton Friedman 109, 113, 121, 125

Mincer Equation 125

mineral resources $30,75,77$

Ministry of Environmental

Protection 169, 175, 177

mixed economy $29,37,38$

modern political systems 66

monetary expansion 64

monetary policy $32,40,63,65$,

$68,125,143$

Montreal Protocol 103, 169

moral judgment 6,72

multinational agreement $\quad 104$

National Climate Change Program 179
National Development and Reform Commission (NDRC) 29-32, 34-37, 40, 90, 155, $157,170,171$

national income identity 183

National Leading Group for Dealing with Climate Change 179

National People's Congress 30, $40,50,61,66,155,172$

National Political Consultative Conference 50

National Working Group for Dealing with Climate Change 179

Nationalist government 64

Naughton, Barry 80

neocolonialism in Africa 76

New Delhi 83

New York Times 5, 12, 67, 75, $77,83,139,173$

news media 40

nickel 79

Niu Linlin 199

non-government employee 48

Non-government school 58

non-state enterprise $30,36,128$, 147,149

North Africa 32, 76

North America 17, 18

nuclear energy 5,30

numerical targets 29, 32

NYSE Composite Index 202

observation $19,48,60,89,189$, $192,193,202$

old-age insurance system 54

one-child policy 47

one-party system $\quad 49,67,68$ 
Organization for Economic

Cooperation and Development (OECD) 59

outflow of capital 75,81

output target 45

overseas Chinese $42,51,58,59$

panel data regressions 113

Paraguay 59

parameter stability 115

patent 5,96

Peking University 17

penalty provisions 62

Pentagon 12

People's Bank 9, 40, 56, 65,

161,162

permanent income hypothesis

$113,183,184,186,189$

petroleum 30

"phony capitalism" 114

physical capital 11, 21, 22, 200

planned economy $3,29,30,85$

Planning Commission 17, 29-31, 40

Political Bureau 18

political freedom $7,45,47,50$, 51,66

Political stability $\quad 4,84$

pollution

air pollution $102,103,167$, 168, 174-176

water pollution 102,103 , $167-169,177$

pollution control 104

population

aging population 165,166

Prasad, Eswar 80

present value theory 113 price

constant price $183,184,190$

dual price system 128

equilibrium price 176

housing price 133

index price $8,64,65,101$, $144,161,184,185$

price control 8,161

price level $8,9,64,65,68$, $113,126,161$

price of stocks 113, 195-197, 201,202

private enterprises $42,43,45$, $47-49,53,84,89-91,114$

private farming $46,48,100$

private hospital 101

Product Quality Law 61-63

productive efficiency 155

profit

illegal profit seeking 6

public assets 157,158

public opinion 67

public participation 67

purchasing power parity 4,85 , 129

qualitative knowledge 116

quality control systems 61,62

quantity theory of money 64

quotas of output 46

\section{R\&D 32}

railroads 30,141

Rajan, Raghuram G. 80

random examination 61

random walk with drift 183

rate of change $113,184,186$,

196, 202 
rate of depreciation $183,184,186$ rate of interest 10,162

rate of return $11,77,125,196$,

$$
197,201-205
$$

rationing $46-48$

raw material 118,128

recession $10,19,29,32,133$,

$143,144,163,164$

Record of the Historian 75

Red Guards 16

regional differences 32

regression $113,184,189,192$,

193, 199-201, 204, 205, 209

religious freedom 51

Renewable Energy Law 180

Renminbi 64

residential housing 118

Reuters 9, 161

risk taking $6,7,26,43,72,73$, 144

RMB undervaluation 9,162

role of economists 39

role of government $45,48,52,68$

role of planning 29

ruled by law $6,27,72$

ruled by people $6,27,72$

rural markets 46,48

rural population $53-55,97-100$, 125,140

rural poverty $97,98,105,109$, 123

Russia $\quad 75,85-87$

SARS (Severe Acute Respiratory

Syndrome) 67

school 43, 53, 56-58, 60, 68,

$80,98,99,102,109,148$,

$149,152,153,174,210$ school voucher 102

security $32,48,50,53,54,68$, $80,84,134$

"Seek Truth from Facts" 47, 127

service industries 47

service sector $31,33,34$,

Shang Dynasty 22

Shanghai 17, 42, 90, 102, 113, 118,125

Shanghai Composite Index 202

share-holding corporations 47

Shi Zhengrong 20,91

Serven, Luis 80

shock treatment 119

Sima Qian 22, 75

Sims, Christopher 122

Singapore $3,16,22,46$

Smith, Adam 107

$\mathrm{SO}_{2}$ srubber 174,175

social instability $7,38,73,97$, 105

social security system $\quad 54,68$

social status 27 ,

social welfare $37,45,53,68,140$

socialist economy 36,50

solar panels $5,20,173,174$

solid biomass 179

Solid Waste Law 103, 168

Song Dynast 22

South America 11, 81

South China sea 12

South Korea 3, 16, 22, 46, 84, 86,102

Southwest University of Finance and Economics 159

Soviet bloc countries 46

Soviet Union $15,16,55,119$, 127 
Soviet-style central economic planning 45

standard error 184,192

Standing Committee 18

State Commission for

Reconstructing the Economic

System 17, 118

State Council 8, 15, 17, 29,

34-36, 40, 55, 61, 62, 101,

161,169

State Development Planning

Commission 30

State Economic and Trade

Commission (SETC) 30

State Economic Commission 40 state enterprises $16,30,46,47$, $54,68,100,112,118,119$, $127,128,147,149,155$,

157

State Environmental Protection

Administration (SEPA) 169

State Food and Drug

Administration 62

State Planning Commission 29,

31,40

state-space framework 205

stimulus package $189,192,193$

stochastic volatility model 201 stocks

A shares 200

B shares 200

stock exchanges

New York 113, 201, 205

Shanghai 113, 200, 201, 205

Shenzhen 200

stock markets $47,195,196,197$,

$199,200,201$

stock option 26 stock prices

co-movements 195, 196, 199, 201

New York 199

Shanghai 195, 209

sulfur dioxide 103, 167, 171

Suntech 20,91, 173

supervision $35,61,62,67,175$

t-statistics 204, 206

Taiwan $3,16,21,22,46,84,86$, 89-92, 111, 114, 117, 118, $120,121,123,126,148$

Tanzania-Zambia Railway 76

tariff 47

technology $5,11,12,31,43,79$, $83,114,128,170,176,178$, 180,200

telecommunications 30

territorial rights 12

textile 30

theoretical economics 108

Three-farm Program 97

thrift $6,72,138$

Tiananmen 65

Tiger, Mr. 20

time-varying regression

time-varying coefficient 205 , 206, 208, 209

time-varying parameter 201, 205

total expenditure $60,112,125$

total output $5,34,43,86,112$,

129

trade

domestic trade 47

trade surplus 9, 115, 162, 163

trading partners 46 
transportation $30,84,138$

travel $46,47,128,138,148$

Tsiang, S.C. 117,121

tuition $59,60,68,98,99,122$, 125,148

Tunisia 76

two stage least squares 184 , 192

two-tier price system $\quad 118$

unemployment insurance system 54

unemployment rate 33,133 , 143

United Nations Educational, Scientific and Cultural

Organization (UNESCO) 59 United Nations Guidelines for

Consumer Protection 62

United Nations Population

Division 165, 166

university administrator 147 , 148,152

urban and rural construction 30 urban housing $112,128,134$, 144

urban industrial reform 47 urbanization rate 33

US $3-13,19,26,27,37,38,41$, $42,71-73,75-77,80,81,85$, $102,107-110,118,129,133$, $134,144,152,153,162-164$, $170,171,173,178,180,196$, 200,210

US financial institutions $5,7,73$

US GDP 4,5

US government debt 8

US Treasury bonds 10, 162, 164
VAR 113,126

Vietnam 12, 27

village Communist Party secretaries 98

volatility $196,201,202,203$

Wang Chuanfu 20

Wang Yanan Institute 194, 196, 199

Wang Yongching 21,91

Wang Zijun 69

war against terrorism $\quad 7,76$

War with Japan 22

"Washington Consensus" 7, 38

Water and Soil Conservation Law 103,168

water beds 102, 167

Water Pollution Prevention and

Control Law 103, 168

water resources 30

welfare benefits 97,140

Wen Jiabao 76, 103, 169, 170, 177

Western countries 46

western development 10, 109, 163

Western Europe 17,102

Western Pacific 12, 13

working environment 27

working ethics 27

"Working Group of Five" 120

World Bank 5, 80

World Expo in Shanghai 168

World Health Organization

(WHO) 102, 167

World Trade Organization 47

World War II 3, 12, 22, 109, 114

WTO membership 66 
Xu Yuan 174

Yangtze River 102, 167

Yao Yilin 17

Yellow River 102, 167
Zhao Ziyang 17, 118

Zhong Yongguang 159

Zhongshan University 18

Zhu Rongji 65

Zimbabwe 59 